\title{
Computational prediction and experimental validation of evolutionarily conserved microRNA target genes in bilaterian animals
}

\author{
Kahori Takane ${ }^{1,2}$, Kosuke Fujishima ${ }^{1,2}$, Yuka Watanabe ${ }^{1,2}$, Asako Sato ${ }^{1}$, Nobuto Saito ${ }^{1,2}$, Masaru Tomita ${ }^{1,2}$, \\ Akio Kanai ${ }^{1,2^{*}}$
}

\begin{abstract}
Background: In many eukaryotes, microRNAs (miRNAs) bind to complementary sites in the $3^{\prime}$-untranslated regions (3'-UTRs) of target messenger RNAs (mRNAs) and regulate their expression at the stage of translation. Recent studies have revealed that many miRNAs are evolutionarily conserved; however, the evolution of their target genes has yet to be systematically characterized. We sought to elucidate a set of conserved miRNA/target-gene pairs and to analyse the mechanism underlying miRNA-mediated gene regulation in the early stage of bilaterian evolution.
\end{abstract}

Results: Initially, we extracted five evolutionarily conserved miRNAs (let-7, miR-1, miR-124, miR-125/lin-4, and miR-34) among five diverse bilaterian animals. Subsequently, we designed a procedure to predict evolutionarily conserved miRNA/target-gene pairs by introducing orthologous gene information. As a result, we extracted 31 orthologous miRNA/target-gene pairs that were conserved among at least four diverse bilaterian animals; the prediction set showed prominent enrichment of orthologous miRNA/target-gene pairs that were verified experimentally. Approximately $84 \%$ of the target genes were regulated by three miRNAs (let-7, miR-1, and miR-124) and their function was classified mainly into the following categories: development, muscle formation, cell adhesion, and gene regulation. We used a reporter gene assay to experimentally verify the downregulation of six candidate pairs (out of six tested pairs) in HeLa cells.

Conclusions: The application of our new method enables the identification of 31 miRNA/target-gene pairs that were expected to have been regulated from the era of the common bilaterian ancestor. The downregulation of all six candidate pairs suggests that orthologous information contributed to the elucidation of the primordial set of genes that has been regulated by miRNAs; it was also an efficient tool for the elimination of false positives from the predicted candidates. In conclusion, our study identified potentially important miRNA-target pairs that were evolutionarily conserved throughout diverse bilaterian animals and that may provide new insights into early-stage miRNA functions.

\section{Background}

MicroRNAs (miRNAs) are a class of short (18-25 nucleotides) non-coding RNAs that regulate gene expression posttranscriptionally. Their regulatory potential relies heavily on the recognition of binding sites that are located mainly in the 3'-untranslated regions (3'-UTRs) of target messenger RNAs (mRNAs) [1]. Currently, numerous miRNAs with diverse sequences are being characterized in a

\footnotetext{
* Correspondence: akio@sfc.keio.ac.jp
${ }^{1}$ Institute for Advanced Biosciences, Keio University, Tsuruoka 997-0017,

* Correspondence: akio@sfc.keio.ac.jp
${ }^{1}$ Institute for Advanced Biosciences, Keio University, Tsuruoka 997-0017, Japan
}

(c) 2010 Takane et al; licensee BioMed Central Ltd. This is an Open Access article distributed under the terms of the Creative Commons Attribution License (http://creativecommons.org/licenses/by/2.0), which permits unrestricted use, distribution, and reproduction in any medium, provided the original work is properly cited. wide range of species [2], suggesting that this small RNA molecule has a major effect on phylogeny. The importance of miRNAs is also suggested from recent research demonstrating that miRNA-guided gene regulation is involved in diverse biological functions, such as cell differentiation, development, carcinogenesis, and tumour suppression [3-6]. For example, phylogenetically conserved miRNAs (e. g., let-7, miR-1, miR-124, and miR-125) are involved in cell differentiation and development [7-10]. In this case, let-7 regulates the expression of $R A S$ proteins known as critical oncogene products [11]. Moreover, miR-34, another evolutionarily conserved miRNA, is a direct downregulator of 
p53 and is involved in a genetic pathway that promotes cell-cycle progression [12].

In recent years, more than 700 miRNAs have been identified in humans [13], and this number is increasing. In a recent report by Friedman et al., the expression of a large number of target genes is predicted to be regulated by miRNAs [14]; however, relatively few of these have been verified experimentally. To overcome this problem, a series of computational methods has been developed to predict a large number of miRNA targets; e.g., TargetScan [14], RNAhybrid [15], MicroTar [16], PITA [17], miRanda [18], and PicTar [19]. Nevertheless, these computational approaches often provide numerous target candidates with a large number of false positives because of the weak complementarity between miRNAs and 3'-UTRs [20]. Recently, a phylogenetic profiling approach has been applied to overcome this limitation. For example, studies of the evolution of orthologous target sites have provided insights into the prediction of efficient miRNA targets [21]. As for miRNAs, many miRNA families are found among various bilaterian animals, suggesting that several miRNAs and their target genes may have co-evolved; however, these features have yet to be systematically characterized.

In this study, we hypothesized that the core regulatory relationship between miRNAs and their target genes were conserved throughout the evolution of bilaterian animals. In addition, by predicting these relationships, we sought to elucidate the core function of miRNAs in the primitive gene-regulatory network of the common bilaterian ancestor. Accordingly, we focused on five miRNAs (let-7, miR-1, miR-124, miR-125/lin-4, and miR-34) that are conserved among bilaterian species (Homo sapiens (H. sapiens), Mus musculus (M. musculus), Gallus gallus (G. gallus), Drosophila melanogaster (D. melanogaster), and Caenorhabditis elegans (C. elegans)) and designed a procedure to extract conserved miRNA/target-gene pairs. We extracted evolutionarily conserved miRNA/targetgene pairs based on hybridization patterns and orthologous information. In addition, we experimentally verified several candidate pairs to support our methodology. Our results suggest a functional role of three major miRNAs (let-7, $m i R-1$, and $m i R-124)$ that regulated genes related to development, muscle formation, and cell adhesion. These results suggest a new role for the core function of miRNAs in the primitive gene-regulatory network of the common bilaterian ancestor.

\section{Results and Discussion}

\section{Extraction of evolutionarily conserved miRNAs among} five bilaterian animals

To extract conserved miRNA/target-gene pairs, we chose five model species (H. sapiens, M. musculus, G. gallus, D. melanogaster, and C. elegans) among bilaterian animals, for which there exists a vast array of data on both miRNAs and mRNAs [13]. Previously, several important features were described to classify miRNAs into families. It is well known that the seed sequence (the 5' side of the miRNA sequence) is important for interaction with the target mRNAs [22]. Many miRNA target prediction software programs were developed using the features of seed sequences [14-19]. Moreover, several features have been proposed to identify conserved miRNA families, such as conservation of the mature miRNA sequence (features of the earliest miRNA classification in miRBase) [23] and information on the phylogenetic relationship among miRNAs [24]. By focusing on these features, we proposed the following criteria for extracting well-conserved miRNA families among five species: (1) complete seed sequence matching, (2) mature miRNA sequence identity exceeding $75 \%$, and (3) high conservation among miRNA families, considering the phylogenetic relationship among miRNAs (category I) [24]. Consequently, from 2,404 mature miRNA sequences, we extracted five miRNA families (let-7, miR-1, miR-124, miR-125/lin-4, and miR-34) conserved evolutionarily among the five bilaterian animals (Table 1). The sequence identity among most of the conserved miRNA families was over $80 \%$. In particular, the sequences of let-7 and $m i R-1$ family members showed very high mature miRNA sequence identity (exceeding 90\%) among all five bilaterian species, which suggests that a strong selective pressure exists for the nucleotide sequence. Huang et al. described the extraction of 15 conserved miRNA families among six bilaterian animals (H. sapiens, M. musculus, G. gallus, D. rerio, D. melanogaster, and C. elegans) based on their original classification method [24], which included our five miRNA families. In this manuscript, we devised more stringent criteria based on nucleotide conservation to extract highly conserved miRNA families. Nematostella vectensis and Amphimedon queenslandica, which diverged before the emergence of bilaterian animals, reportedly express various types of miRNAs [25]; however, none of these miRNAs are sequentially similar to the five evolutionarily conserved miRNA families found in the current study (data not shown), which suggests that these evolutionarily conserved miRNAs appeared after the divergence of bilaterian animals or were lost in $N$. vectensis and $A$. queenslandica.

\section{Filtering and enrichment of the evolutionarily conserved miRNA/target-gene pairs}

To extract genes targeted through evolution by the five conserved miRNAs, we designed a procedure that comprised three screening steps (Figure 1 and Table 2). In step 1 , we extracted potential target genes based on 
Table 1 List of miRNAs conserved among various bilaterian animals

\begin{tabular}{ccccccc}
\hline \multicolumn{7}{c}{ Species } \\
\hline miRNA family & H. sapiens & M. musculus & G. gallus & D. melanogaster & C. elegans & Identity (\%) \\
\hline let-7 & let-7a & let-7a & let-7a & let-7 & let-7 \\
miR-1 & miR-1 & miR-1 & miR-1a & miR-1 & miR-1 & 95.5 \\
miR-124 & miR-124 & miR-124 & miR-124a & miR-124 & miR-124 & 82.6 \\
miR-125/lin-4 & miR-125b & miR-125b-5p & miR-125b & miR-125 & lin-4 & 81.8 \\
miR-34 & miR-34a & miR-34a & miR-34a & miR-34 & miR-34 & 79.2 \\
\hline
\end{tabular}

optimal free-energy information with a requirement of complete seed-sequence-matching using the RNAhybrid software [15], which predicts potential binding sites of short RNAs among target sequences. For this purpose, the optimal free energy was determined beforehand to efficiently cover experimentally validated miRNA/targetgene pairs. We calculated the free energy of 139 experimentally validated miRNA/target-gene pairs with a complete match of seed sequence, as assessed using RNAhybrid; subsequently, we defined the optimal free energy as $<-17 \mathrm{kcal} /$ mole of potential miRNA/targetgene pairs (data not shown). The evaluation of targetgene extraction during each step was carried out using an index termed "Enrichment" (see Methods section). As a result, in step 1, the number of potential targetgene candidates decreased from 357,430 to 153,387 , with an enrichment index of 1.8. This prediction set contained 112 of the 145 experimentally validated target-gene pairs.

In step 2, we considered the 3'-UTR binding pattern of the miRNA/target mRNA. We defined four binding parameters (i.e., number of mismatches of mRNA within the whole miRNA sequence, number of mismatches of miRNA within the whole miRNA sequence, number of G-U wobble pairs within the whole miRNA sequence, and number of G-U wobble pairs within the seed sequence) of the hybridization pattern (Additional file 1) for optimisation of the thresholds for each of the features used to predict reliable miRNA/mRNA pairs. The ranges of the four binding parameters were determined by calculating the coverage of 112 miRNA-mRNA pairs verified experimentally and of 153,387 miRNA-mRNA pairs predicted for each binding feature (Figure 2A-D). Five hundred parameter combinations were plotted on a 2D graph, using "Enrichment" on the $\mathrm{X}$-axis and "Ratio of experimentally verified miRNA/mRNA" on the Y-axis (Figure 2E). From these parameter combinations, we defined the optimal combination of binding parameters for efficient screening based on a maximum EC value of 184.7 (number of mismatches of mRNA within the whole miRNA sequence: 12; number of mismatches of miRNA within the whole miRNA sequence: 10 ; number of G-U wobble pairs within the whole miRNA sequence: 4; and number of G-U wobble pairs within the seed sequence: 0 ). Accordingly, the number of potential target-gene candidates was reduced from 153,387 to 28,201 and the experimentally validated target genes decreased from 112 to 76 after introduction of the criterion of optimal hybridization pattern (Enrichment index, 6.6) (Table 2).

Finally, in step 3, we incorporated orthologous gene information and extracted genes that were evolutionarily conserved among more than four diverse bilaterian animals, including $H$. sapiens. As a result, the number of predicted miRNA/target-gene pairs was minimized substantially, from 10,356 to 31 , using a significantly high Enrichment index of 25.7 (Table 2). The number of predicted miRNA/target-gene pairs was especially high for

Table 2 Summary of target gene extraction after each screening step

\begin{tabular}{|c|c|c|c|c|c|c|c|c|}
\hline & $\begin{array}{c}H . \\
\text { sapiens }\end{array}$ & $\begin{array}{c}\text { M. } \\
\text { musculus }\end{array}$ & $\begin{array}{c}G . \\
\text { gallus }\end{array}$ & $\begin{array}{c}D . \\
\text { melanogaster }\end{array}$ & $\begin{array}{c}\text { C. } \\
\text { elegans }\end{array}$ & $\begin{array}{c}\text { Ratio of predicted } \\
\text { miRNA/target-gene } \\
\text { pairs (\%) }\end{array}$ & $\begin{array}{c}\text { Ratio of experimentally } \\
\text { verified miRNA/target-gene } \\
\text { pairs }(\%)\end{array}$ & Enrichment \\
\hline $\begin{array}{c}\text { All possible } \\
\text { miRNA/gene pairs }\end{array}$ & 97820 & 98790 & 53995 & 52850 & 53975 & $357430 / 357430(100)$ & $145 / 145(100)$ & 1.0 \\
\hline $\begin{array}{l}\text { STEP } 1 \text { (Free } \\
\text { energy and seed } \\
\text { region) }\end{array}$ & 56666 & 53420 & 20410 & 14098 & 8793 & $153387 / 357430(42.9)$ & $112 / 145(77.2)$ & 1.8 \\
\hline $\begin{array}{l}\text { STEP } 2 \text { (Optimal } \\
\text { parameter) }\end{array}$ & 12405 & 11256 & 2524 & 1118 & 898 & 28201/357430 (7.9) & 76/145 (52.4) & 6.6 \\
\hline STEP 3 & & & 31 & & & $31 / 10356^{*}(0.3)$ & $4 / 52(7.7)$ & 25.7 \\
\hline
\end{tabular}

The total number of possible target genes for the five miRNAs (let-7, miR-1, miR-124, miR-125/lin-4, and miR-34) is represented for the five model species at each screening step (see Figure 1). Asterisks indicate the number of orthologous genes conserved among at least four species. Enrichment was calculated as the "ratio of experimentally verified miRNA/target-gene pairs" divided by the "ratio of predicted miRNA/target-gene pairs". 


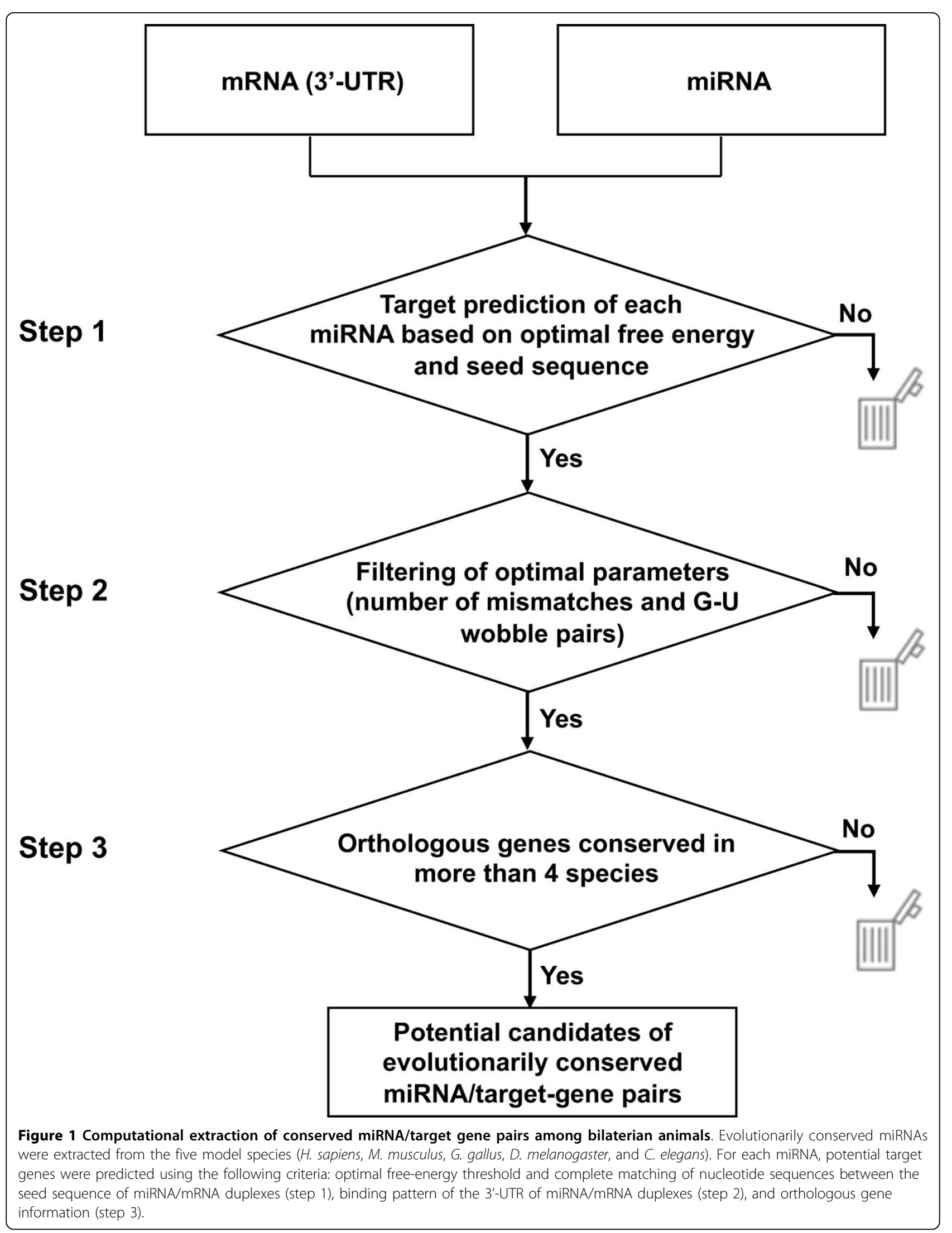




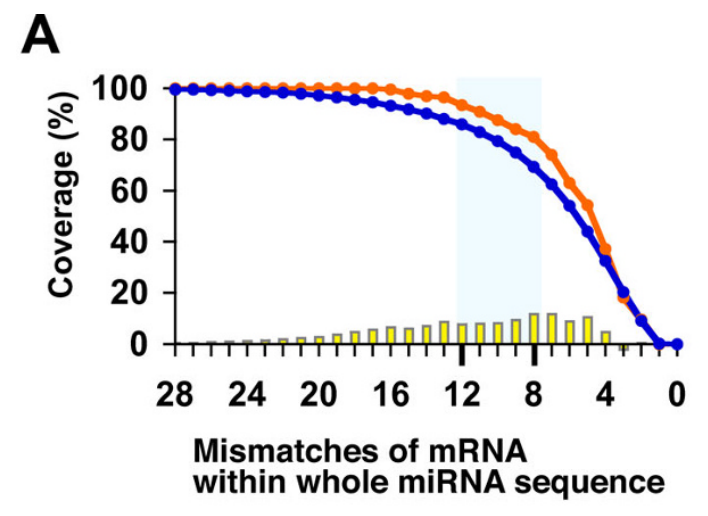

C

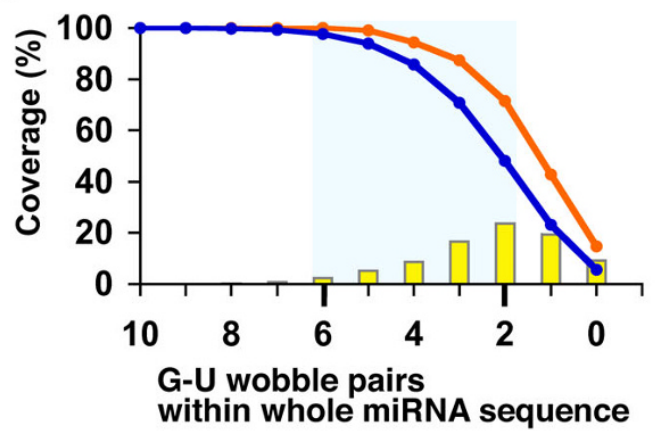

B

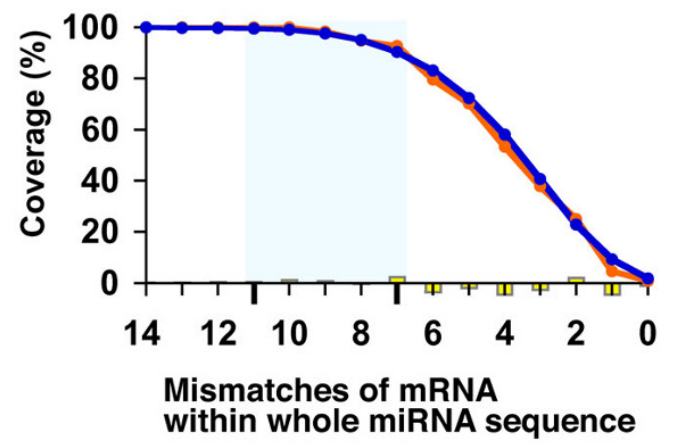

D

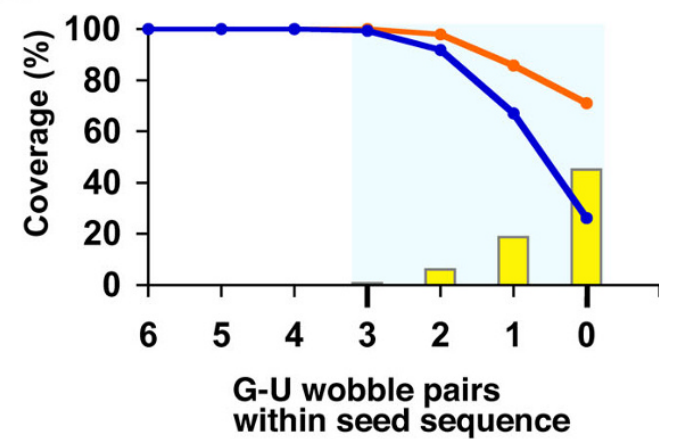

E

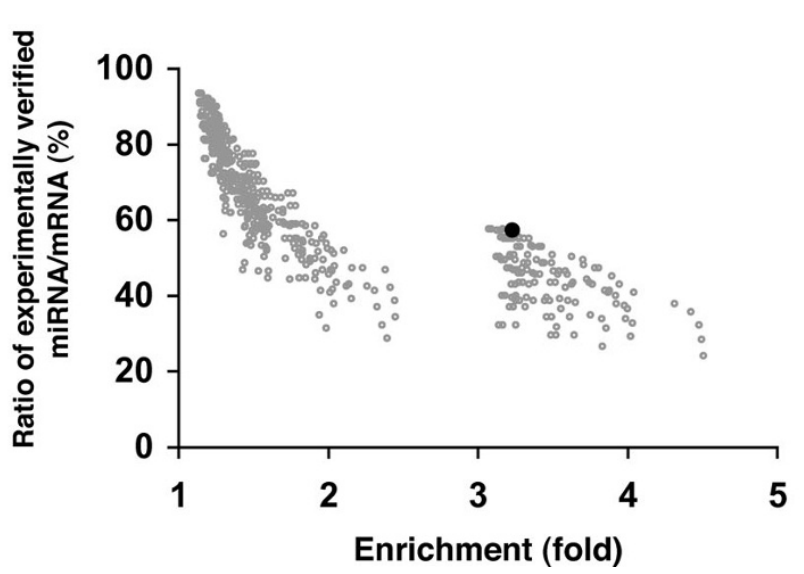

Figure 2 Parameters used for the prediction of miRNA/mRNA pairs and their coverage. To optimize the binding parameters of miRNA/ mRNA duplexes, we determined the coverage of four binding parameters (mismatch of mRNA within the whole miRNA sequence (A), mismatch of miRNA within the whole miRNA sequence $(B), G-U$ wobble pairs within the whole miRNA sequence $(C)$, and $G-U$ wobble pairs within the seed sequence (D)). Calculation of the coverage was performed using 112 experimentally verified miRNA/mRNA pairs (orange line) and 153,387 predicted miRNA/mRNA pairs (blue line). The yellow bar indicates differences in coverage between experimentally verified and computationally predicted miRNA/mRNA pairs. Four or five points chosen from the highest yellow bar were used as the range of each of the four binding parameters (blue squares) used in this study. We determined parameter space using the binding patterns of the miRNA/mRNA pairs based on four features (E). Five hundred parameter combinations were plotted on a 2D graph using "ratio of experimentally verified miRNA/mRNA" on the Y-axis and "Enrichment" on the X-axis. Black circles (57.3\% of the coverage and 3.2-fold of the Enrichment) indicate the point that corresponded to optimized parameters for the prediction of final conserved miRNA/target pairs: 12 for the number of mismatches in the mRNA, 10 for the number of mismatches in the miRNA, 4 for the number of $\mathrm{G}-U$ wobble pairs within the whole miRNA sequence, and 0 for the number of $\mathrm{G}-\mathrm{U}$ wobble pairs within the seed sequence (see Methods section). 
the three miRNAs let-7 (eight targets), $m i R-1$ (seven targets), and $m i R-124$ (eleven targets) compared with $m i R$ 125/lin-4 (three targets) and miR-34 (two targets) (Additional file 2). This suggests that let-7, $m i R-1$, and $m i R-$ 124 may have played a major role in primordial miRNA gene regulation in the common bilaterian ancestor. To verify the significance of conserved miRNA-target gene pairs, we performed same sequence analysis (from step 1 to step 3) against total 25 species-specific miRNAs (5 miRNAs each from 5 species) as a control experiment supposing that these miRNAs are also conserved in other bilaterians. For example, target prediction of hsamiR-2277, a species-specific miRNA in human was performed in all 5 species (step 1 and step 2) and conserved targets were extracted (step 3 ). As a result, 11 out of 25 non-conserved miRNAs did not show any conserved miRNA-target gene pair. Furthermore, average number of the miRNA-target gene pairs of the negative control was 2.4 , which is statistically lower than that of conserved miRNA-target gene pairs 6.2 based on the Welch's t-test $(\mathrm{P}<0.05)$. These results support that number of genes achieved from the prediction of conserved miRNAs target genes in this study is indeed significant. In summary, we developed a new filtering method for extracting evolutionarily conserved miRNA/ target-gene pairs, which was used to extract 31 reliable miRNA/target-gene pairs among the five families of miRNAs.

We discovered that only one orthologous target gene, calponin-3 (CNN3), was conserved completely among the five bilaterian animals (Figure 3A). As for target genes conserved in four species, we found, for example, the La-related protein 4 (LARP4), ETS domain-containing protein Elk-3 (ELK3), argonaute-4 (EIF2C4), transgelin-2 (TAGLN2), and V-type proton ATPase subunit B brain isoform (ATP6V1B2) genes (Figure 3B and Additional file 3 ). Of note, the same approximate position of the predicted target site was observed in the orthologous 3'-UTR of CNN3 (120 nucleotides (nt)), LARP4 (3,100 nt), EIF2C4 (220 nt), and TAGLN2 (50 nt) among vertebrates (Figure 3 and Additional file 3). According to Bartel et al., the distribution of miRNA target sites within the 3'-UTR is biased near the mRNA stop codon or poly-A tail compared with the middle portion of 3'UTR [26]. Our results show that target site distribution varied according to the type of miRNA target gene. The target sites on 3'-UTR of CNN3 and TAGLN2 were biased near the stop codon, from $H$. sapiens to $C$. elegans (Figure 3A and Additional file 3). Regarding the other candidates, we observed all types of target site distribution on 3'-UTR. A future statistical analysis of miRNA target-site distribution among conserved miRNA/target-gene pairs is required to substantiate this view. With the exception of 3'-UTR of the LARP4 gene, most of the binding patterns of evolutionarily conserved target sites were sequentially different, without taking the seed region into consideration. The target-site binding patterns within 3'-UTR of the LARP4 gene were identical between $H$. sapiens and $M$. musculus (Figure 3B), although the similarity of the two 3'-UTR sequences was $\sim 70 \%$ (data not shown). A recent study reported on cooperative regulation by multiple miRNAs [19]. Likewise, the band 4.1-like protein 4B (EPB41L4B) gene was an orthologous target of two different types of miRNAs: $m i R-1$ and $m i R-124$ (Table 3 ). Our analysis suggests that multiple miRNA regulation may have already existed in the era of ancestral bilaterian species.

\section{Experimental validation of miRNA target genes}

To validate the evolutionarily conserved miRNA/targetgene candidates, we performed transfection and luciferase assays on 6 of the 31 identified evolutionarily conserved miRNA/target-gene candidates. Four of the six candidates (CNN3, LARP4, TAGLN2, and ATP6V1B2) were predicted to be regulated by $m i R-1$, while one candidate (ELK3) was predicted to be targeted by $m i R-124$, and the final candidate (EIF2C4) was predicted to be the target of let-7. We used the well-established downregulation of the delta-like protein 1 precursor $(D L L 1)$ gene by $m i R-34$ as a positive control [22], and the LARP4/let7 pair, which was extracted up to step 2 in our analysis, was chosen as a non-evolutionarily conserved pair. We subcloned the 3'-UTR sequence downstream from the Renilla luciferase gene (Figure 4A) and co-transfected $100 \mathrm{ng}$ of the 3'-UTR reporter construct into HeLa cells using 5, 20, and 60 pmol of miRNA Mimics (hsa-let-7a, hsa-miR-1, hsa-miR-124, hsa-miR-34a, and miRIDIAN microRNA Hairpin Inhibitor Negative Control \#1) (see Methods section). We observed the downregulation of six out of six candidates and of the positive control compared with the negative controls (Figure 4B, a-g). Typical results of the reporter gene assay are shown in Figure $4 \mathrm{~B}$ for the indicated amounts of miRNAs $(5,20$, and $60 \mathrm{pmol})$. The downregulation of these candidates was significant $(P<0.01)$, although some of these pairs represented an inhibition of only $30 \%$ under the current conditions. Among these candidates, TAGLN2 was previously suggested to be downregulated by $m i R-1$, as assessed by microarray analysis [27]. This feature was recently confirmed using the "pulsed stable isotope labelling with amino acids in cell culture" (pSILAC) method and a reporter gene assay [28]. Regarding the let-7/LARP4 combination, the expression of LARP4 was not downregulated after let-7 transfection, which was supported statistically (Figure 4B, h). These experimental results suggest that our new method has the potential for efficiently extracting reliable miRNA/target-gene pairs and may be effective in the elucidation of the 
Table 3 Evolutionarily conserved genes regulated by miRNAs

\begin{tabular}{|c|c|c|c|c|c|c|c|}
\hline \multicolumn{3}{|r|}{ Target gene } & \multirow[b]{2}{*}{$\begin{array}{l}\text { let- } \\
7\end{array}$} & \multirow[b]{2}{*}{$\begin{array}{l}\operatorname{miR}- \\
1\end{array}$} & \multirow[b]{2}{*}{$\begin{array}{l}\operatorname{miR}- \\
124\end{array}$} & \multirow[b]{2}{*}{$\begin{array}{l}\operatorname{miR}-125 / \\
\text { lin4 }\end{array}$} & \multirow[b]{2}{*}{$\begin{array}{l}\operatorname{miR}- \\
34\end{array}$} \\
\hline ID & Name & Function & & & & & \\
\hline \multicolumn{8}{|c|}{ Candidates targeted by multiple miRNAs } \\
\hline ENSG00000095203 & EPB41L4B & Band 4.1-like protein $4 \mathrm{~B}$ & & $+^{* *}$ & + & & \\
\hline \multicolumn{8}{|c|}{ Candidates targeted by single miRNAs } \\
\hline ENSG00000187772 & LIN28B & Lin-28 homolog B & + & & & & \\
\hline ENSG00000198799 & LRIG2 & $\begin{array}{l}\text { Leucine-rich repeats and immunoglobulin-like domains protein } 2 \\
\text { precursor }\end{array}$ & + & & & & \\
\hline ENSG00000086544 & ITPKC & Inositol-trisphosphate 3-kinase C & + & & & & \\
\hline ENSG00000196233 & LCOR & Ligand-dependent corepressor & + & & & & \\
\hline ENSG00000139263 & LRIG3 & $\begin{array}{l}\text { Leucine-rich repeats and immunoglobulin-like domains protein } 3 \\
\text { precursor }\end{array}$ & + & & & & \\
\hline ENSG00000134698 & $\mathrm{EIF} 2 \mathrm{C} 4$ & Eukaryotic translation initiation factor $2 \mathrm{C} 4$ & + & & & & \\
\hline ENSG00000170456 & DENND5B & MGC24039 protein & + & & & & \\
\hline ENSG00000136231 & IGF2BP3 & Insulin-like growth factor 2 mRNA-binding protein 3 & + & & & & \\
\hline ENSG00000158710 & TAGLN2 & Transgelin-2 & & $+^{*}$ & & & \\
\hline ENSG00000143549 & TPM3 & Tropomyosin alpha-3 chain & & $+^{*}$ & & & \\
\hline ENSG00000117519 & CNN3 & Calponin-3 & & + & & & \\
\hline ENSG00000071073 & MGAT4A & $\begin{array}{l}\text { Alpha-1,3-mannosyl-glycoprotein 4-beta- } \mathrm{N} \text { - } \\
\text { acetylglucosaminyltransferase A }\end{array}$ & & + & & & \\
\hline ENSG00000161813 & LARP4 & La-related protein 4 & & + & & & \\
\hline ENSG00000147416 & ATP6V1B2 & Vacuolar ATP synthase subunit B, brain isoform & & + & & & \\
\hline ENSG00000135862 & LAMC1 & Laminin subunit gamma-1 precursor & & & $+^{*}$ & & \\
\hline ENSG00000125695 & STRADA & STE20-related adapter protein & & & + & & \\
\hline ENSG00000131459 & GFPT2 & Glucosamine-fructose-6-phosphate aminotransferase & & & + & & \\
\hline ENSG00000111145 & ELK3 & ETS domain-containing protein Elk-3 & & & + & & \\
\hline ENSG00000151726 & ACSL1 & Long-chain-fatty-acid-CoA ligase 1 & & & + & & \\
\hline ENSG00000164144 & ARFIP1 & Arfaptin-1 & & & $t^{* *}$ & & \\
\hline ENSG00000080819 & CPOX & Coproporphyrinogen III oxidase, mitochondrial precursor & & & + & & \\
\hline ENSG00000138279 & ANXA7 & Annexin A7 & & & + & & \\
\hline ENSG00000150093 & ITGB1 & Integrin beta-1 precursor & & & $t^{* *}$ & & \\
\hline ENSG00000093167 & LRRFIP2 & Leucine-rich repeat flightless-interacting protein 2 & & & + & & \\
\hline ENSG00000116141 & MARK1 & Serine/threonine-protein kinase MARK1 & & & & + & \\
\hline ENSG00000166797 & FAM96A & Protein FAM96A & & & & + & \\
\hline ENSG00000131914 & LIN28 & Lin-28 homolog A & & & & $+^{*}$ & \\
\hline ENSG00000113282 & CLINT1 & EPN4_HUMAN Isoform 2 of Q14677 - Homo sapiens & & & & & + \\
\hline ENSG00000137872 & SEMA6D & Semaphorin-6D precursor & & & & & + \\
\hline
\end{tabular}

primordial regulatory relationships between miRNAs and their target genes during the early stage of bilaterian evolution.

\section{Possible regulation of evolutionarily conserved miRNA targets in bilaterian animals}

To provide further insight into the primary functions of evolutionarily conserved miRNAs (Table 3 ), we next focused on the functions of the target genes and found that evolutionarily conserved miRNA/target genes could be largely classified into four functional categories: development, differentiation, muscle movement, and gene regulation. First, we describe the function of evolutionarily conserved genes involved in development and differentiation. The laminin subunit gamma-1 precursor (LAMC1) gene, which was possibly regulated by $m i R-$ 124 , is one of the major components of the basement membrane. According to Smyth et al. [29], null mutation of $L A M C 1$ causes embryonic lethality because of the absence of the basement membrane and failure to differentiate the endoderm. Among other candidates regulated by $m i R-124$, the expression of the leucine-rich repeat flightless-interacting protein 2 (LRRFIP2) gene induces an extra axis in Xenopus laevis embryos [30]. 


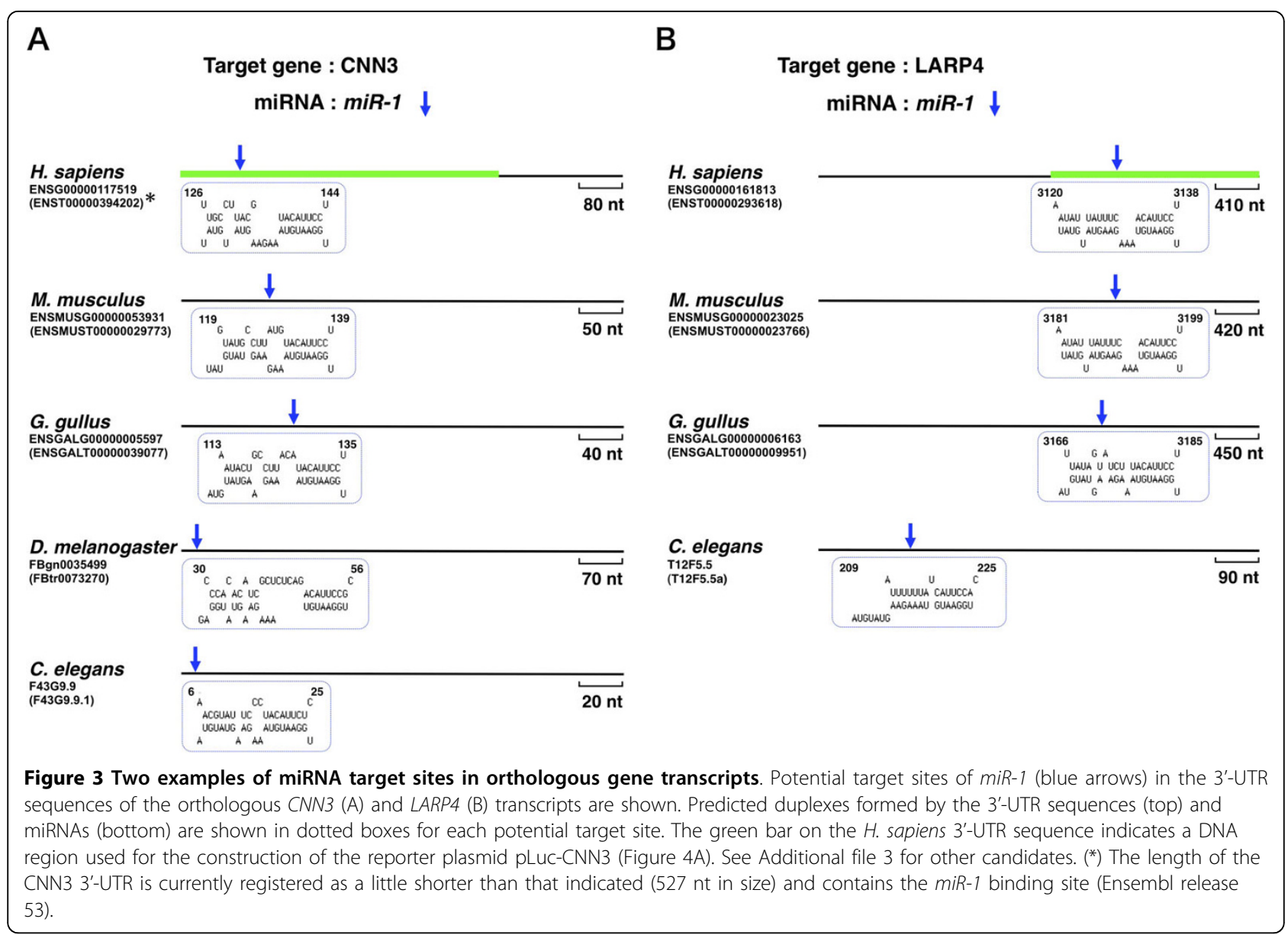

Moreover, the semaphorin-6D precursor (SEMA6D) gene, plays an important role in cardiac morphogenesis during chick embryonic dev which was predicted as a candidate of miR-34 targeting, elopment [31]. The lin28 gene, which regulates developmental timing in C. elegans, is reportedly controlled by lin- 4 , as assessed using in vivo experiments [32]. Subsequently, the lin-28 gene was also found to be regulated by $m i R-125$, which is an orthologous miRNA of lin-4, in H. sapiens and M. musculus [33]. Our prediction confirmed the regulation of orthologous lin-28 genes by lin-4/miR-125 miRNA in $H$. sapiens, $M$. musculus, and C. elegans and further suggested that a similar regulatory relationship was conserved in G. gallus. Interestingly, our prediction showed that the orthologous let-7/lin-28 pair was also evolutionarily conserved among bilaterians. This feature had been validated experimentally in $H$. sapiens [34]; however, in C. elegans, lin-28 is expressed in an early stage of development, while let-7 is expressed in a later stage of development, suggesting that regulation of $\operatorname{lin}-28$ by let-7 is subtle in C. elegans [7]. It would be of interest to analyse the interaction of the let-7/lin-28 pair in other species, such as M. musculus and G. gallus, to understand whether stage-specific expression of let-7 was present in the common ancestor of bilaterian animals or if it is a trait acquired later during evolution, as many of the evolutionarily conserved miRNA/targetgene pairs were related to an essential function involved in differentiation and development.

Next, we focused on the tissue-specific miRNA/target genes. Among the 30 evolutionarily conserved target candidates, approximately one-third were expressed in a tissue-specific manner in humans, according to the BioGPS portal http://biogps.gnf.org. miR-1 is highly expressed in muscle tissues [8]. Here, three candidate genes regulated by $m i R-1$ (i.e., TAGLN2, CNN3, and TPM3) are also expressed in muscle tissues, according to BioGPS. TAGLN2 is a homolog of TAGLN, which encodes an actin-binding protein and is a diagnostic marker of breast and colon carcinoma in humans [35]. The CNN3 gene also encodes an actin-binding protein that represses bone morphogenetic protein (BMP) signalling in chondrocytes, which is important for bone formation [36]. Moreover, the TPM3 gene encodes yet another actin-binding protein that modulates muscle contraction. The other miRNA, miR-124, is expressed in 
A

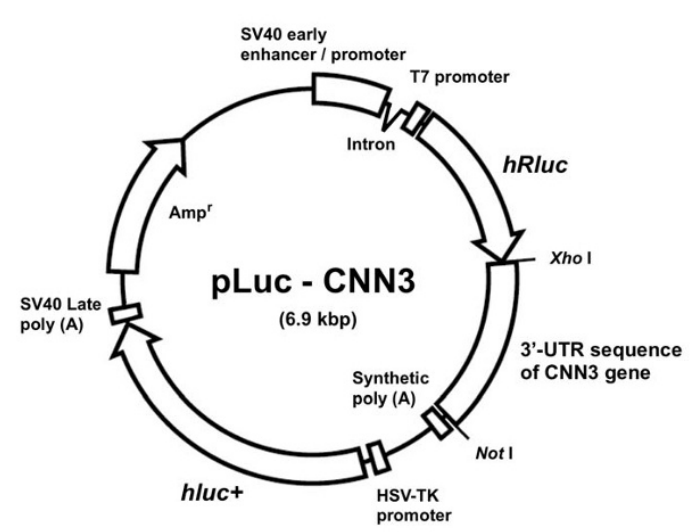

B

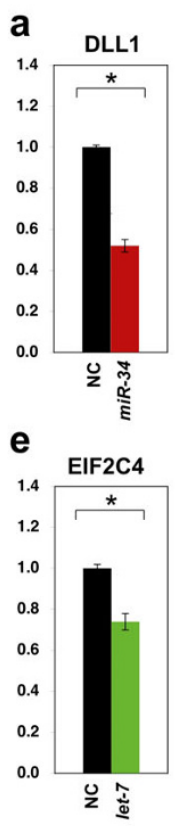

\section{b}

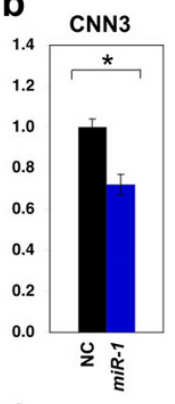

f TAGLN2

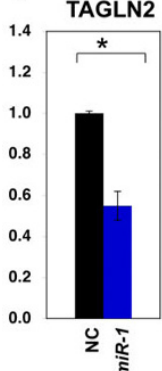

C

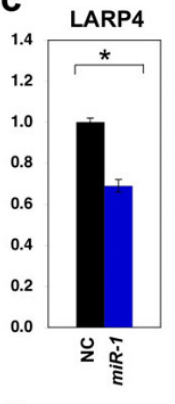

$\mathbf{g}_{1.4}$ ATP6V1B2

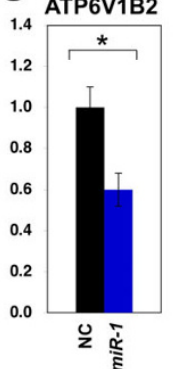

d
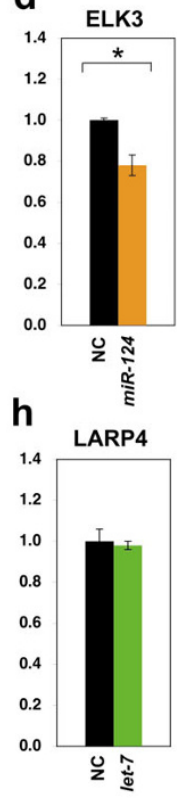

Figure 4 Example of the 3'-UTR reporter plasmid and experimental validation. The 3'-UTR sequences of DLL1, CNN3, LARP4, ELK3, EIF2C4, TAGLN2, and ATP6V1B2 were subcloned into the Xhol/Notl site of the psiCHECK ${ }^{\mathrm{TM}}-2$ vector. CNN3 was chosen as representative of the eight candidates listed above (see Methods section). (B) HeLa cells were cotransfected with each combination of $100 \mathrm{ng}$ of reporter plasmid and the indicated amounts of each miRNA (DLL1, 5 pmol of miR-34 (a); CNN3, 60 pmol of miR-1 (b); LARP4, 20 pmol of miR-1 (c); ELK3, 60 pmol of miR124 (d); EIF2C4, 60 pmol of let-7 (e), TAGLN2, 5 pmol of miR-1 (f), ATP6VIV2, 60 pmol of miR-1 (g), and LARP4, 60 pmol of let-7 (h)). Colours depict each miRNA: miR-34 (red), miR-1 (blue), miR-124 (orange), let-7 (green), and negative control (black). The relative expression of the luciferase gene was measured $24 \mathrm{~h}$ after transfection. The normalized luciferase activity of the control vector was set as 1.0. The data represent the average of three experiments and SDs. ${ }^{*} P<0.01$.

the nervous system [9]. Similarly, one of the evolutionarily conserved target candidates, the glucosamine-fructose-6-phosphate aminotransferase 2 (GFPT2) gene, is expressed in the central nervous system [37]. In addition, the annexin A7 (ANXA7) gene, which is another candidate target of miR-124, is involved in the development of the murine brain [38]. The striking overlap between the tissue specificity of evolutionarily conserved miRNA and that of their target genes suggests that one of the main functions of primordial miRNAs may have been the regulation of genes implicated in the temporary control of the development of muscle and of the nervous system, in a tissue-specific manner.

Finally, we found two interesting candidate genes, EIF2C4 and LARP4, which encode translation-related proteins. It is well accepted that miRNAs are regulators of gene expression, mostly at the translational level [1]. EIF2C4 is also known as Argonaute 4 (AGO4). Although the function of AGO4 is unknown, other Argonaute protein family members are involved in the RNAinduced silencing complex (RISC), which is essential for the miRNA or siRNA pathways. A previous microarray analysis performed in HepG2 cells revealed that the EIF2C4 gene was affected by let-7 [6]. In the present study, we demonstrated for the first time the direct downregulation of EIF2C4 by let-7, as assessed using a reporter gene assay in HeLa cells (Figure 4B, e); therefore, we speculate that negative-feedback regulation of EIF2C4 by let-7 exists in the miRNA pathway. It has been reported that regulation of the AGO1 mRNA, which is a major component of the RISC complex, in the miRNA pathway by miR-168 controls plant development in Arabidopsis thaliana [39]. Another candidate, $L A R P 4$, encodes a member of La-motif protein family that controls translational efficiency [40]. We also demonstrated the downregulation of the LARP4 gene via $m i R-1$ using a reporter gene assay (Figure $4 \mathrm{~B}, \mathrm{c}$ ), which further supports our contention that some of the evolutionarily conserved miRNAs may play an important role in the regulation of translation by controlling the expression levels of translation factors and by negatively regulating their own miRNA pathway.

\section{Conclusions}

We developed a procedure to extract potential evolutionarily conserved miRNA/target-gene pairs based on orthologous gene information from five bilaterian animals, and efficiently extracted 31 evolutionarily 
conserved miRNA/target-gene pairs from 357,430 pairs. We experimentally validated the downregulation of six candidate pairs (out of six tested pairs) in HeLa cells, which suggests that our method using orthologous information was efficient in extracting evolutionarily conserved miRNA target-gene candidates. Our findings reveal that miRNA target sites were conserved among various species, and demonstrate that especially let-7-, miR-1-, and miR-124-mediated gene regulation may have played an important role throughout evolution, in processes such as development, differentiation, and muscle movement. Moreover, our results indicate that miRNA-mediated translational regulation as well as tissue-specific expression of miRNA/target-gene pairs may have already existed in the common bilaterian ancestor. In conclusion, our study will provide new insights into the early stages of miRNA function.

\section{Methods}

\section{miRNA and 3'-UTR sequence data}

We downloaded 2,404 mature miRNA sequences (885 for H. sapiens, 689 for M. musculus, 520 for G. gallus, 153 for D. melanogaster, and 157 for C. elegans) from the miRBase, version $13.0 \mathrm{http}: / /$ microrna.sanger.ac.uk/ sequences/[13]. We downloaded sequences corresponding to 3'-UTR using the Ensembl transcript ID annotation in FASTA format $(40,498$ transcripts for H. sapiens, 3,332 for M. musculus, 13,089 for G. gallus, 16,822 for D. melanogaster, and 13,560 for C. elegans) from the Ensmart database Ensembl release 53 http://www. ensembl.org/index.html[41]. Orthologous gene information was also downloaded from the Ensmart database Ensembl release 53. We obtained 145 experimentally verified miRNA/target-gene pairs from TarBase Version 5.0.1 http://diana.cslab.ece.ntua.gr/tarbase/[42].

\section{Identification of miRNAs conserved among bilaterian animals}

The 2,404 miRNA sequences were aligned using ClustalX [43] with the following alignment parameters: gap opening, 22.50; gap extension, 0.83; and bootstrap value, 100 . We checked the conservation of 2,404 miRNA sequences to extract evolutionarily conserved miRNAs. We defined the conservation threshold as an "overall sequence identity > $75 \%$ with complete matching of the seed sequence (1-7, 2-8, or 3-9 nucleotides from the miRNA's 5' end)"; furthermore, we introduced information on the phylogenetic relationship among miRNAs to extract reliably conserved miRNAs and used highly conserved miRNA families (category I) [24].

\section{Extraction of evolutionarily conserved miRNA/target-gene pairs among bilaterian animals}

To extract evolutionarily conserved miRNA/target-gene pairs among bilaterian animals, we devised a three-step filtering approach (Figure 1). In step 1, we predicted genes targeted by each of the five miRNAs (let-7, miR-1, miR-124, miR-125/lin-4, and miR-34) using RNAhybrid, which is fast and flexible software for miRNA target prediction, with the free-energy option and the seedsequence option [15]. The RNA duplex free-energy filter was defined as the appropriate value that led to the efficient extraction of experimentally verified miRNA/target-gene pairs. We also considered a complete match across the seed sequence $(1-7,2-8$, or 3-9 nucleotides from the miRNA's 5' end), which was used as a filter by adding the seed option of RNAhybrid.

In step 2, we used four binding parameters of the hybridization pattern of the miRNA/mRNA duplexes. According to a recent study, a binding rule is likely to exist for the recognition of target mRNAs by miRNAs [34]. Moreover, G-U wobble pairs within miRNA/ mRNA duplexes play a key role in the interaction with target mRNAs [44]. Subsequently, potential candidates were extracted using four binding parameters (number of mismatches of mRNA within the whole miRNA sequence, number of mismatches of miRNA within the whole miRNA sequence, number of G-U wobble pairs within the whole miRNA sequence, and number of G-U wobble pairs within the seed sequence) of the miRNA/ mRNA duplexes (Additional file 1). We used the hybridization pattern of experimentally verified and predicted miRNA/mRNA pairs to calculate coverage, by changing these binding parameters one by one (28-0 for the number of mismatches of mRNA within the whole miRNA sequence; 14-0 for the number of mismatches of miRNA within the whole miRNA sequence; $10-0$ for the number of G-U wobble pairs within the whole miRNA sequence; and 6-0 for the number of G-U wobble pairs within the seed sequence) (Figure 2A-D). The range of each of the four binding parameters was determined based on the coverage of experimentally verified and predicted miRNA/mRNA pairs. Parameter combinations were then plotted on a $2 \mathrm{D}$ graph by calculating the "ratio of experimentally verified miRNA/mRNA" and "Enrichment" using the points in the four parameter ranges (Figure 2E). The criterion "Enrichment" was defined and calculated as the value of "ratio of experimentally verified miRNA/target-gene pairs" divided by the "ratio of predicted miRNA/target-gene pairs". We obtained the most effective combination of four binding parameters for extracting miRNA/target-gene pairs based on the EC value. Parameter conbination with highest EC value was selected.

In step 3, orthologous gene information was used to extract orthologous genes targeted by the same type of miRNA. For the retrieval of evolutionarily conserved miRNA/target-gene pairs from various bilaterian animals, we set the orthologous gene information criteria 
as orthologous genes conserved in at least four species, each containing the miRNA target site of interest.

\section{Expression vectors}

To construct target-site reporter plasmids, each DNA fragment (3'-UTR sequence of the DLL1 gene (668 nt; accession no. AF003522), ELK3 gene (519 nt; accession no. BC017371), EIF2C4 gene (2148 nt; accession no. AB046787), TAGLN2 gene (1391 nt; accession no. D21261), LARP4 gene (1678 nt; accession no. AY004310), CNN3 gene (1391 nt; accession no. BC025372), and ATP6V1B2 gene (1208 nt; accession no. L35249)) was amplified from HeLa genomic DNA via polymerase chain reaction using site-specific primers and was inserted into the XhoI/NotI sites of the psiCHECK-2 plasmid vector (which encodes both firefly and Renilla luciferases; Promega, Madison, WI, USA) (Figure 4A). The oligonucleotide was designed to introduce XhoI and NotI sites at the 5' and 3' termini, respectively. The resulting plasmids were termed pLucDLL1, pLuc-CNN3, pLuc-LARP4, pLuc-ELK3, pLucEIF2C4, pLuc-TAGLN2, and pLuc-ATP6V1B2, respectively. miRIDIAN ${ }^{\mathrm{mm}}$ miRNA Mimic for hsa-let-7a, hsamiR-1, hsa-miR-124, hsa-miR-34a, and negative control (miRIDIAN microRNA Hairpin Inhibitor Negative Control \#1) were purchased from Dharmacon. miRNA Mimic molecules are chemically modified doublestranded RNA oligonucleotides.

The sequences of the oligodeoxyribonucleotides used for PCR were as follows:

(1) DLL1_S: 5'-TTACTCGAGAATGGAAGTGA GATGGCAAGAC-3'

(2) DLLl_A: 5'-TTAGCGGCCGCTTGTCATTCA TAAAATTTATTT-3'

(3) CNN3_S: 5'-TTACTCGAGTCCACACAGAAG GAGCTCAG-3'

(4) CNN3_A: 5' -TTAGCGGCCGCCAGGAAGAG CAAATGCATCA-3'

(5) LARP4_S: 5'-TTACTCGAGGTTCCCATTT GATGGCATGT-3'

(6) LARP4_A: 5'-TTAGCGGCCGCCATAG CACCTTGGCGATGTT-3'

(7) ELK3_S: 5'-TTACTCGAGCGTCTGGCCACAAT TAAGGA-3'

(8) ELK3_A: 5'-TTAGCGGCCGCTGCTTTCA TATTGCCCACTG-3'

(9) EIF2C4_S: 5'-TTACTCGAGTTCTACCAG CAGCTCGGAAT-3'

(10) EIF2C4_A: 5'-TTAGCGGCCGCTTGGATTC CAGCAAGTCCTC-3'

(11) TAGLN2_S: 5'-TTACTCGAGCCCTCCCAC GAATGGTTAAT-3'

(12) TAGLN2_A: 5'-TTAGCGGCCGCATGGAAAAT GAGAAGCCACG-3'
(13) ATP6V1B2_S: 5'-TTACTCGAGTCCGCGCTCTT GTGAAATAC-3'

(14) ATP6V1B2_A: 5'-TTAGCGGCCGCATAAT CATGCTGACTCCCCC-3'

\section{Transfection and luciferase reporter assay}

Transient transfection and luciferase assays were performed as described previously, with slight modifications [22]. Briefly, HeLa cells were grown in $10 \%$ FBS in DMEM and seeded in 24-well plates $24 \mathrm{~h}$ before transfection. Cells were transfected with the indicated amounts of reporter and miRNA Mimic (100 ng of target reporter and 5, 20, and 60 pmol of miRNA Mimic) in the presence of Lipofectamine 2000 (Invitrogen, Carlsbad, CA, USA). Firefly and Renilla luciferase activities were measured consecutively using the Dual-luciferase assay system (Promega) $24 \mathrm{~h}$ after transfection, according to the manufacturer's instructions.

\section{List of abbreviations used}

miRNA: microRNA; 3'-UTR: 3'-untranslated region; EC value: multiplied value of Enrichment and Coverage; nt: nucleotides; SD: standard deviation.

\footnotetext{
Additional file 1: Basic concept of miRNA/mRNA duplex formation.

An example of the binding pattern of miRNA (bottom)/mRNA (top) duplexes is shown using cel-let-7 and die-1 3'-UTR sequences from C. elegans. The green and blue squares depict mismatched nucleotide sequences of the mRNA and miRNA, respectively. The red square depicts $\mathrm{G}-U$ wobble pairs within the whole miRNA sequence and the black arrow pinpoints a $\mathrm{G}-U$ wobble pair within the seed sequence. Click here for file

[http://www.biomedcentral.com/content/supplementary/1471-2164-11101-S1.PDF ]

Additional file 2: Summary of the number of target genes in each extraction step

Click here for file

[http://www.biomedcentral.com/content/supplementary/1471-2164-11101-S2.PDF ]

Additional file 3: Additional examples of miRNA target sites in orthologous gene transcripts used for experimental verification. Potential target sites of miR-124 (orange arrows) in the 3'-UTR sequences of orthologous ELK3 transcripts (A). Potential target sites of let-7 (green arrows) in the $3^{\prime}$-UTR sequences of orthologous EIF2C4 transcripts (B). Potential target sites of miR-1 (blue arrows) in the $3^{\prime}-U T R$ sequences of orthologous TAGLN2 transcripts (C) and ATP6V1B2 transcripts (D). 3'-UTR sequences and miRNAs are shown in dotted boxes for each potential target site; the colours of dotted boxes and arrows correspond to those of each miRNA.

Click here for file

[http://www.biomedcentral.com/content/supplementary/1471-2164-11101-S3.PDF ]
}

\section{Acknowledgements}

We are grateful to Mizuki Komasa, Motomu Matsui, and Hikaru Taniguchi (Keio University, Japan) for useful discussions. This research was supported in part by a Grant-in-Aid from the 21st Century Centre of Excellence (COE) Program entitled "Understanding and Control of Life's Function via Systems Biology" (Keio University); research funds from the Yamagata Prefectural Government and Tsuruoka City in Japan; and a grant from Keio University. 


\section{Author details}

'Institute for Advanced Biosciences, Keio University, Tsuruoka 997-0017, Japan. ${ }^{2}$ Systems Biology Program, Graduate School of Media and Governance, Keio University, Fujisawa 252-8520, Japan.

\section{Authors' contributions}

KT participated in all aspects of the study. KF contributed to the overall conception of this work and performed the functional profiling of miRNAtargeted genes. YW supported the design of the target-gene extraction procedure and validated new miRNA target candidates using transfection and luciferase reporter assay experiments. AS constructed the experimental system. NS participated in the validation of new miRNA target candidates using transfection and luciferase reporter assay experiments. MT supervised the project. AK participated in the experimental design and drafted the manuscript. All authors read and approved the final manuscript.

Received: 27 October 2009

Accepted: 9 February 2010 Published: 9 February 2010

\section{References}

1. Bartel DP: MicroRNAs: genomics, biogenesis, mechanism, and function. Cell 2004, 116(2):281-297

2. Pasquinelli AE, Reinhart BJ, Slack F, Martindale MQ, Kuroda MI, Maller B, Hayward DC, Ball EE, Degnan B, Muller P, et al: Conservation of the sequence and temporal expression of let-7 heterochronic regulatory RNA. Nature 2000, 408(6808):86-89.

3. Shivdasani RA: MicroRNAs: regulators of gene expression and cell differentiation. Blood 2006, 108(12):3646-3653.

4. Wienholds E, Plasterk RH: MicroRNA function in animal development. FEBS Lett 2005, 579(26):5911-5922.

5. He L, Thomson JM, Hemann MT, Hernando-Monge E, Mu D, Goodson S, Powers S, Cordon-Cardo C, Lowe SW, Hannon GJ, et al: A microRNA polycistron as a potential human oncogene. Nature 2005, 435(7043):828-833.

6. Johnson CD, Esquela-Kerscher A, Stefani G, Byrom M, Kelnar K, Ovcharenko D, Wilson M, Wang X, Shelton J, Shingara J, et al: The let-7 microRNA represses cell proliferation pathways in human cells. Cancer Res 2007, 67(16):7713-7722.

7. Reinhart BJ, Slack FJ, Basson M, Pasquinelli AE, Bettinger JC, Rougvie AE, Horvitz HR, Ruvkun G: The 21-nucleotide let-7 RNA regulates developmental timing in Caenorhabditis elegans. Nature 2000, 403(6772):901-906

8. Chen JF, Mandel EM, Thomson JM, Wu Q, Callis TE, Hammond SM, Conlon FL, Wang DZ: The role of microRNA-1 and microRNA-133 in skeletal muscle proliferation and differentiation. Nat Genet 2006, 38(2):228-233.

9. Yu JY, Chung KH, Deo M, Thompson RC, Turner DL: MicroRNA miR-124 regulates neurite outgrowth during neuronal differentiation. Exp Cell Res 2008, 314(14):2618-2633.

10. Caygill EE, Johnston LA: Temporal regulation of metamorphic processes in Drosophila by the let-7 and miR-125 heterochronic microRNAs. Curr Biol 2008, 18(13):943-950

11. Johnson SM, Grosshans H, Shingara J, Byrom M, Jarvis R, Cheng A, Labourier E, Reinert KL, Brown D, Slack FJ: RAS is regulated by the let-7 microRNA family. Cell 2005, 120(5):635-647.

12. He L, He X, Lim LP, de Stanchina E, Xuan Z, Liang Y, Xue W, Zender L, Magnus J, Ridzon D, et al: A microRNA component of the p53 tumour suppressor network. Nature 2007, 447(7148):1130-1134.

13. Griffiths-Jones S, Saini HK, van Dongen S, Enright AJ: miRBase: tools for microRNA genomics. Nucleic Acids Res 2008, , 36 Database: D154-158

14. Friedman RC, Farh KK, Burge CB, Bartel DP: Most mammalian mRNAs are conserved targets of microRNAs. Genome Res 2009, 19(1):92-105.

15. Rehmsmeier M, Steffen P, Hochsmann M, Giegerich R: Fast and effective prediction of microRNA/target duplexes. RNA 2004, 10(10):1507-1517.

16. Thadani R, Tammi MT: MicroTar: predicting microRNA targets from RNA duplexes. BMC Bioinformatics 2006, 7(Suppl 5):S20.

17. Kertesz M, lovino N, Unnerstall U, Gaul U, Segal E: The role of site accessibility in microRNA target recognition. Nat Genet 2007, 39(10):1278-1284.

18. John B, Enright AJ, Aravin A, Tuschl T, Sander C, Marks DS: Human MicroRNA targets. PLoS Biol 2004, 2(11):e363.
19. Krek A, Grun D, Poy MN, Wolf R, Rosenberg L, Epstein EJ, MacMenamin P, da Piedade I, Gunsalus KC, Stoffel M, et al: Combinatorial microRNA target predictions. Nat Genet 2005, 37(5):495-500.

20. Watanabe $Y$, Tomita M, Kanai A: Computational methods for microRNA target prediction. Methods Enzymol 2007, 427:65-86.

21. Gaidatzis D, van Nimwegen E, Hausser J, Zavolan M: Inference of miRNA targets using evolutionary conservation and pathway analysis. $B M C$ Bioinformatics 2007, 8:69.

22. Lewis BP, Shih IH, Jones-Rhoades MW, Bartel DP, Burge CB: Prediction of mammalian microRNA targets. Cell 2003, 115(7):787-798.

23. Griffiths-Jones S: The microRNA Registry. Nucleic Acids Res 2004, , 32 Database: D109-111.

24. Huang $Y, G u X:$ A bootstrap based analysis pipeline for efficient classification of phylogenetically related animal miRNAs. BMC Genomics 2007, 8:66.

25. Grimson A, Srivastava M, Fahey B, Woodcroft BJ, Chiang HR, King N, Degnan BM, Rokhsar DS, Bartel DP: Early origins and evolution of microRNAs and Piwi-interacting RNAs in animals. Nature 2008, 455(7217):1193-1197.

26. Grimson A, Farh KK, Johnston WK, Garrett-Engele P, Lim LP, Bartel DP: MicroRNA targeting specificity in mammals: determinants beyond seed pairing. Mol Cell 2007, 27(1):91-105.

27. Lim LP, Lau NC, Garrett-Engele P, Grimson A, Schelter JM, Castle J, Bartel DP, Linsley PS, Johnson JM: Microarray analysis shows that some microRNAs downregulate large numbers of target mRNAs. Nature 2005, 433(7027):769-773.

28. Selbach M, Schwanhausser B, Thierfelder N, Fang Z, Khanin R, Rajewsky N: Widespread changes in protein synthesis induced by microRNAs. Nature 2008, 455(7209):58-63.

29. Smyth N, Vatansever HS, Murray P, Meyer M, Frie C, Paulsson M, Edgar D: Absence of basement membranes after targeting the LAMC1 gene results in embryonic lethality due to failure of endoderm differentiation. J Cell Biol 1999, 144(1):151-160.

30. Liu J, Bang AG, Kintner C, Orth AP, Chanda SK, Ding S, Schultz PG Identification of the Wnt signaling activator leucine-rich repeat in Flightless interaction protein 2 by a genome-wide functional analysis. Proc Natl Acad Sci USA 2005, 102(6):1927-1932.

31. Toyofuku T, Zhang H, Kumanogoh A, Takegahara N, Suto F, Kamei J, Aoki K Yabuki M, Hori M, Fujisawa H, et al: Dual roles of Sema6D in cardiac morphogenesis through region-specific association of its receptor, Plexin-A1, with off-track and vascular endothelial growth factor receptor type 2. Genes Dev 2004, 18(4):435-447.

32. Moss $E G$, Lee RC, Ambros V: The cold shock domain protein LIN-28 controls developmental timing in C. elegans and is regulated by the lin4 RNA. Cell 1997, 88(5):637-646.

33. Wu L, Belasco JG: Micro-RNA regulation of the mammalian lin-28 gene during neuronal differentiation of embryonal carcinoma cells. Mol Cell Biol 2005, 25(21):9198-9208.

34. Kiriakidou M, Nelson PT, Kouranov A, Fitziev P, Bouyioukos C, Mourelatos Z, Hatzigeorgiou A: A combined computational-experimental approach predicts human microRNA targets. Genes Dev 2004, 18(10):1165-1178.

35. Shields JM, Rogers-Graham K, Der CJ: Loss of transgelin in breast and colon tumors and in RIE-1 cells by Ras deregulation of gene expression through Raf-independent pathways. J Biol Chem 2002, 277(12):9790-9799.

36. Haag J, Aigner T: Identification of calponin 3 as a novel Smad-binding modulator of BMP signaling expressed in cartilage. Exp Cell Res 2007, 313(16):3386-3394.

37. Oki T, Yamazaki K, Kuromitsu J, Okada M, Tanaka I: cDNA cloning and mapping of a novel subtype of glutamine:fructose-6-phosphate amidotransferase (GFAT2) in human and mouse. Genomics 1999, 57(2):227-234

38. Rick M, Ramos Garrido SI, Herr C, Thal DR, Noegel AA, Clemen CS: Nuclear localization of Annexin A7 during murine brain development. BMC Neurosci 2005, 6:25.

39. Vaucheret $H$, Vazquez $F$, Crete P, Bartel DP: The action of ARGONAUTE1 in the miRNA pathway and its regulation by the miRNA pathway are crucial for plant development. Genes Dev 2004, 18(10):1187-1197.

40. Bousquet-Antonelli C, Deragon JM: A comprehensive analysis of the Lamotif protein superfamily. RNA 2009, 15(5):750-764.

41. Kasprzyk A, Keefe D, Smedley D, London D, Spooner W, Melsopp C, Hammond M, Rocca-Serra P, Cox T, Birney E: EnsMart: a generic system for 
fast and flexible access to biological data. Genome Res 2004,

14(1):160-169.

42. Papadopoulos GL, Reczko M, Simossis VA, Sethupathy P, Hatzigeorgiou AG The database of experimentally supported targets: a functional update of TarBase. Nucleic Acids Res 2009, 37 Database: D155-158.

43. Larkin MA, Blackshields G, Brown NP, Chenna R, McGettigan PA, McWilliam H, Valentin F, Wallace IM, Wilm A, Lopez R, et al: Clustal W and Clustal X version 2.0. Bioinformatics 2007, 23(21):2947-2948.

44. Lai EC: miRNAs: whys and wherefores of miRNA-mediated regulation. Curr Biol 2005, 15(12):R458-460.

doi:10.1186/1471-2164-11-101

Cite this article as: Takane et al: Computational prediction and experimental validation of evolutionarily conserved microRNA target genes in bilaterian animals. BMC Genomics 2010 11:101

Submit your next manuscript to BioMed Central and take full advantage of:

- Convenient online submission

- Thorough peer review

- No space constraints or color figure charges

- Immediate publication on acceptance

- Inclusion in PubMed, CAS, Scopus and Google Scholar

- Research which is freely available for redistribution

Submit your manuscript at www.biomedcentral.com/submit 